\title{
Double Quantum Dots as Detectors of High-Frequency Quantum Noise in Mesoscopic Conductors.
}

\author{
Ramón Aguado ${ }^{1,2}$ and Leo P. Kouwenhoven ${ }^{1}$ \\ 1-Department of Applied Physics and DIMES, Delft University of Technology, P.O.Box 5046, 2600 GA, Delft, The \\ Netherlands. \\ 2-Department of Physics and Astronomy, Rutgers University, Piscataway, NJ 08854-8019, USA.
}

We propose a measurement set-up for detecting quantum noise over a wide frequency range using inelastic transitions in a tunable two-level system as a detector. The frequencyresolving detector consists of a double quantum dot which is capacitively coupled to the leads of a nearby mesoscopic conductor. The inelastic current through the double quantum dot is calculated in response to equilibrium and non-equilibrium current fluctuations in the nearby conductor, including zeropoint fluctuations at very low temperatures. As a specific example, the fluctuations across a quantum point contact are discussed.

Two-level systems (TLS) coupled to a dissipative environment are canonical model systems to study dephasing in quantum mechanics [1]. The reversed problem is a TLS that measures the characteristics of a specific environment. The transition rate for levels separated by an energy $\epsilon$, is a measure of the spectral density of the fluctuations in the environment at a frequency $f=\epsilon / h$. Transitions are allowed when energy can be exchanged with the environment. Recently, two device structures were realized that can be used as tunable TLS. In a superconducting single-electron transistor a Cooper-pair [2] and in a double quantum dot (DQD) an electron [3] can make inelastic transitions between two discrete energy states. In this work we calculate the rate for inelastic transitions in a DQD coupled to an environment formed by a second mesoscopic device.

Small electronic devices have interesting equilibrium and non-equilibrium noise properties which are nonlinear in frequency [4. In equilibrium, a transition occurs going from low-frequencies, where Johnson-Nyquist noise due to thermal fluctuations dominates, to high frequencies where quantum noise due to zero-point fluctuations (ZPF) prevails. When the device is voltage biased, non-equilibrium fluctuations can become dominant. These lead to shot noise in the current, which has been measured near zero-frequency [5] and at several high frequency values where ZPF become dominant. [6] The idea of using a mesoscopic device -quantum point contact (QPC) - as an environment for another device-quantum dot- has successfully been used in the so-called 'whichpath' detector: [7] the dc shot-noise of the QPC modifies the transport properties of the dot, leading to dephasing.
Here we propose a setup for studying the effect of broadband fluctuations on the inelastic rate in a TLS. This setup provides a frequency-resolved detection over a large frequency range of the fluctuations in mesoscopic systems. A wide frequency range requires that the frequency dependent impedance of the whole circuit is taken into account. Below, we first describe the basic properties of a DQD, then formulate transition probabilities in terms of the noise spectrum, followed by calculations where the specific environment is formed by a QPC.

a)
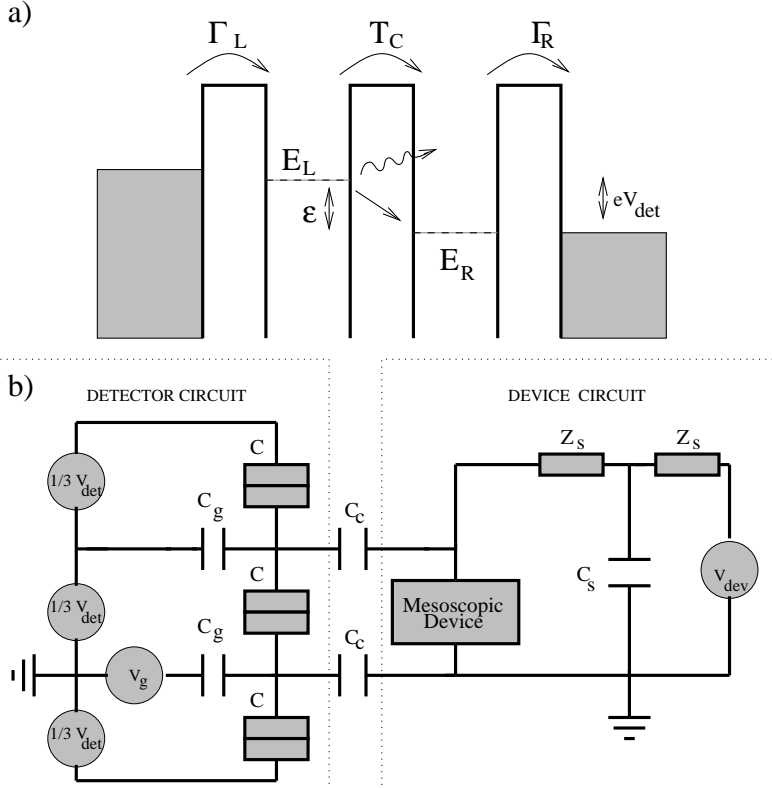

FIG. 1. (a) Energy diagram of a DQD in the regime of high bias voltage. (b) Circuit for capacitively coupling the DQD to a second mesoscopic device, e.g. a QPC. The detector and device circuits are separately biased by different voltages. The symbols $\boxminus$ in the detector correspond to the three tunnel barriers in a DQD.

A DQD is a fully-controllable TLS. The separation between levels $\epsilon \equiv E_{L}-E_{R}$, the tunnel rates across the left and right barriers, $\Gamma_{L}, \Gamma_{R}$, and the tunnel coupling between the dots, $T_{c}$ (see Fig.1a), can be tuned separately by means of gate voltages. If $\epsilon \gg T_{c}$, we can neglect coherence effects due to mixing between $E_{L}$ and $E_{R}$ [8]. Then a non-zero current for $\epsilon \neq 0$ necessarily involves emission $(\epsilon>0)$ or absorption $(\epsilon<0)$ of quanta to or from the environment. An applied bias voltage, $V_{\text {det }}$, shifts the two Fermi levels in the two leads. The 
higher Fermi energy in the left lead allows that for $\epsilon>0$, the high energy state, $E_{L}$, can be occupied by tunneling through the left barrier. An inelastic transition with rate $\Gamma_{i}$, followed by tunneling through the right barrier yields an inelastic current, $I_{\text {inel }}(\epsilon)=\frac{e}{\hbar}\left(\Gamma_{L}^{-1}+\Gamma_{i}^{-1}+\Gamma_{R}^{-1}\right)^{-1}$. When the central tunnel barrier is made the largest, i.e. $\Gamma_{i}<<\Gamma_{L}, \Gamma_{R}$, the inelastic current is governed by time dependent fluctuations $\delta \epsilon(t)=e \delta V(t)$ and can be calculated from perturbation theory on $T_{c}$ : [1, 9 ]

$$
I_{\text {inel }}(\epsilon) \simeq \frac{e}{\hbar} \Gamma_{i}(\epsilon)=\frac{e}{\hbar} T_{c}^{2} P(\epsilon)
$$

$P(\epsilon)$ being the the probability for the exchange of energy quanta with the environment: 10 12]

$$
P(\epsilon)=\frac{1}{2 \pi \hbar} \int_{-\infty}^{\infty} \exp \left[J(t)+i \frac{\epsilon}{\hbar} t\right] d t
$$

All the information about the environment is contained in the autocorrelation of the phase operators $J(t) \equiv$ $\langle[\delta \hat{\phi}(t)-\delta \hat{\phi}(0)] \delta \hat{\phi}(0)\rangle$. [13] $\delta \hat{\phi}(t)=\frac{e}{\hbar} \int^{t} d t^{\prime} \delta \hat{V}\left(t^{\prime}\right)$ are the conjugate phases of the voltage fluctuations $\delta \hat{V}(t)$ (characterized by the spectral density $S_{V}(\omega)$ ). We are interested in current fluctuations, $S_{I}(\omega)$, generated in a nearby mesoscopic device. Fig. 1(b) shows an example of a circuit that capacitively couples a mesoscopic device to a DQD. Both noise spectra are related through: $S_{V}(\omega)=|Z(\omega)|^{2} S_{I}(\omega)(Z(\omega)$ being the trans-impedance connecting detector and device circuits) and then:

$$
J(t)=\frac{2 \pi}{\hbar R_{K}} \int_{-\infty}^{\infty} \frac{|Z(\omega)|^{2}}{\omega^{2}} S_{I}(\omega)\left(e^{-i \omega t}-1\right) d \omega
$$

$R_{K}=h / e^{2} \simeq 25.8 k \Omega$ is the quantum resistance. Importantly, $S_{I}(\omega) \equiv \int_{-\infty}^{\infty} d \tau e^{i \omega \tau}\langle\delta \hat{I}(\tau) \delta \hat{I}(0)\rangle$ appears in a non-symmetrized form. 14] We shall demonstrate that this is crucial to account correctly for ZPF. Eqs. 1-3 relate the inelastic current through the DQD to the noise spectrum of an arbitrary, nearby mesoscopic device. 15

Our problem is now reduced to the determination of $Z(\omega)$ and $S_{I}(\omega)$ for a specific device embedded in a specific circuit. We consider the circuit in Fig. 1b for coupling the current fluctuations from the device via the capacitors $C_{c}$ into the DQD. The DQD is modelled as three tunnel barriers with capacitances $C$ and biased by a voltage $V_{\text {det }}$. The gate voltage, $V_{g}$, controls $\epsilon$. The device is connected to a voltage source, $V_{d e v}$, via leads characterized by the impedances $Z_{s}$ and the capacitor $C_{s}$. For this circuit we obtain for the trans-impedance:

$Z(\omega)=\frac{\alpha_{1} Z_{s}}{\left(\alpha_{2}\left[1+i \omega Z_{s} C_{c}-\frac{1}{\left(2+i \omega Z_{s} C_{S}\right)}\right]-\alpha_{3} i \omega Z_{s} C_{c}\right)}$

with $\alpha_{1}=\frac{C+C_{g}+C_{c}}{C}, \alpha_{2}=\frac{\left(2 C+C_{g}+C_{c}\right)^{2}-C^{2}}{C_{c} C}$ and $\alpha_{3}=$ $\frac{2 C+C_{g}+C_{c}}{C}$. For small $\omega,|Z(\omega)|^{2} \simeq \rho\left[\gamma /\left(\gamma^{2}+\omega^{2}\right]\right.$ with $\rho=$ $\alpha_{1}^{2} Z_{s} /\left[\alpha_{2}\left(\alpha_{2}\left(C_{c}+C_{s}\right)-\alpha_{3} C_{c}\right)\right]$ and $\gamma=\alpha_{2} /\left[Z_{s}\left(\alpha_{2}\left(C_{c}+\right.\right.\right.$
$\left.\left.\left.C_{s}\right)-\alpha_{3} C_{c}\right)\right]$. At $\omega=0$, it reaches the maximum value $|Z(0)|^{2}=\alpha_{1}^{2} / \alpha_{2}^{2} Z_{s}^{2} \equiv \kappa^{2} R_{K}^{2}$ (i.e. Ohmic environment).

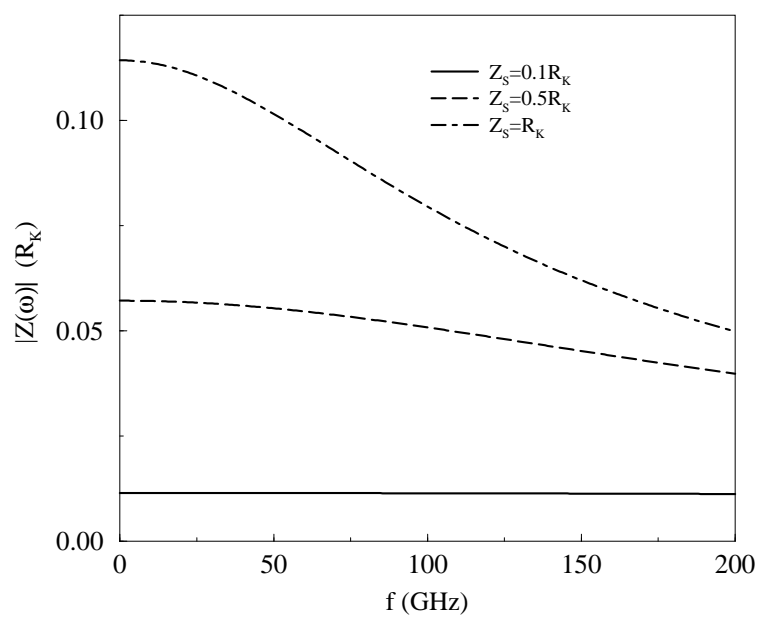

FIG. 2. Frequency dependence of $|Z(\omega)|$ for different Ohmic resistors in the leads. We have taken typical experimental values for: $C=0.05 \mathrm{fF}, C_{g}=C_{c}=0.1 \mathrm{fF}, C_{s}=1 \mathrm{nF}$.

In Fig. 2 we plot $|Z(\omega)|$ for different values of $Z_{s}$ and typical experimental values for the elements in the circuit. For $Z_{s} \rightarrow 0$ the device is shorted (i.e. $Z(\omega) \rightarrow 0$ ) and the detector is insensitive to the fluctuations. For $Z_{s}<0.1 R_{K}$, the trans-impedance is approximately independent of frequency and can be written as $|Z(\omega)|^{2} \simeq$ $|Z(0)|^{2}$. In this case, $I_{\text {inel }}$ is determined only by the frequency dependence of the noise and therefore easier to interpret. If $Z_{s}=0.1 R_{K}$ is taken, then the coupling of the noise into the detector is sufficiently effective. Provided that $J(t)$ does not diverge for long times (see below) we expand $e^{J(t)} \simeq 1+J(t)$ in Eq. 2 and derive:

$$
\begin{aligned}
P(\epsilon) & \simeq\left\{1-\frac{2 \pi}{\hbar R_{K}} \int_{-\infty}^{\infty} d \omega \frac{|Z(\omega)|^{2}}{\omega^{2}} S_{I}(\omega)\right\} \delta(\epsilon) \\
& +\frac{2 \pi}{R_{K}} \frac{|Z(\epsilon / \hbar)|^{2}}{\epsilon^{2}} S_{I}(\epsilon / \hbar) .
\end{aligned}
$$

The first part renormalizes the elastic current (i.e. when $\epsilon=0$ ), which we do not consider further here. Inserting the last term in Eq. 1 we obtain the inelastic current through the DQD detector:

$$
I_{\text {inel }}(\epsilon) \simeq 4 \pi^{2} \kappa^{2} \frac{T_{c}^{2}}{e} \frac{S_{I}(\epsilon / \hbar)}{\epsilon^{2}} .
$$

We note that current fluctuations at frequency $\omega$ result in an inelastic current at level difference $\epsilon=\hbar \omega$. Below we discuss that this detector current is asymmetric in the absorption $(\epsilon<0)$ and emission $(\epsilon>0)$ sides, which results from the asymmetry in the noise due to ZPF.

As an application, we study the current noise spectrum of a QPC. The right quantity to be calculated for our purposes is the non-symmetrized noise. The symmetrized 
version has been calculated in Refs. [16 18]. The time dependent fluctuations of the current around its average are $\delta \hat{I}(\tau)=\hat{I}(\tau)-\langle\hat{I}(\tau)\rangle$, the current operator being $\hat{I}(t)=\frac{2 e}{h} \sum_{\alpha, \beta} \iint_{-\infty}^{\infty} d \epsilon_{1} d \epsilon_{2} I_{\alpha, \beta}\left(\epsilon_{1}, \epsilon_{2}\right) \hat{a}_{\alpha, \epsilon_{1}}^{\dagger}(t) \hat{a}_{\beta, \epsilon_{2}}(t)$ $\left(\hat{a}_{\alpha, \epsilon}^{\dagger}(t)\left[\hat{a}_{\alpha, \epsilon}(t)\right]\right.$ is the creation [annihilation] Heisenberg operator of the scattering state $\psi_{\alpha}(\vec{r}, \epsilon), \alpha \equiv(a, n)$ and $\beta \equiv(b, m)$ represent summations over leads and number of channels and $I_{\alpha, \beta}\left(\epsilon_{1}, \epsilon_{2}\right)$ are the matrix elements of the current with respect to these scattering states). The non-symmetrized noise spectrum can be written as: $S_{I}(\omega) \equiv \int_{-\infty}^{\infty} d \tau e^{i \omega \tau}\langle\delta \hat{I}(\tau) \delta \hat{I}(0)\rangle=$ $\frac{4 e^{2}}{h} \sum_{a, b, n, m} \int d \epsilon I_{a, n, b, m} I_{b, m, a, n} f_{a}(\epsilon)\left[1-f_{b}(\epsilon+\hbar \omega)\right] ; f(\epsilon)$ being the Fermi-Dirac function. If the matrix elements are expressed in terms of energy-independent transmission and reflection scattering matrices, we obtain (see [5] for a complete derivation in the $\omega=0$ limit):

$$
\begin{aligned}
S_{I}(\omega) & =\frac{4}{R_{K}} \sum_{m}^{N} D_{m}\left(1-D_{m}\right)\left\{\frac{\left(e V_{d e v}+\hbar \omega\right)}{1-e^{-\beta\left(e V_{d e v}+\hbar \omega\right)}}\right. \\
& \left.+\frac{\left(\hbar \omega-e V_{d e v}\right)}{1-e^{-\beta\left(\hbar \omega-e V_{d e v}\right)}}\right\}+\frac{4}{R_{K}} \sum_{m}^{N} D_{m}^{2} \frac{2 \hbar \omega}{1-e^{-\beta \hbar \omega}},
\end{aligned}
$$

where $N$ is the number of channels, $D_{m}$ is the transmission probability of the $m^{\text {th }}$ channel, $V_{\text {dev }}$ is the applied voltage and $\beta=1 / k_{B} T$. In equilibrium (i.e. $V_{\text {dev }}=0$ ), we recover the fluctuation-dissipation theorem [19] $S_{I}(\omega)=2 G \frac{2 \hbar \omega}{1-e^{-\beta \hbar \omega}}$, where $G=\frac{2}{R_{K}} \sum_{m}^{N} D_{m}$ is the conductance. Here, our model reduces to the usual theory for the effects of an electromagnetic environment on single electron tunneling [10 12]. Equation (7) is not symmetric $\left(S_{I}(\omega)>S_{I}(-\omega)\right)$ which results from the difference between emission and absorption due to ZPF. This has to be compared with the symmetrized version [16 18] where $S_{I}(\omega)=S_{I}(-\omega)$.

From now on we present calculations for zero temperature. Fig. 3a shows the noise, $S_{I}(\nu)$, vs. the normalized frequency $\nu=\frac{\hbar \omega}{\left|e V_{\text {dev }}\right|}$, for $N=2$ and different values for $D=\sum_{m}^{N} D_{m}$. For $\nu>0$ the noise increases linearly with frequency (with a slope determined by $D$ ) due to ZPF. For $\nu<0$ there are two different cases: for non-open channels the non-equilibrium shot-noise dominates when $-1<\nu<0$, whereas for $\nu<-1$ the noise is zero. For open channels $(D=1$ and $D=2)$ the noise is always zero on the absorption side.

The inset to Fig. 3a shows the voltage dependence of the noise for different values of $\omega$ and $D=1.5$ (see also Ref. [6]). When $\left|e V_{d e v}\right|<\hbar \omega$ the noise spectrum is flat. Here, the fluctuations are dominated by quantum noise and do not change from the equilibrium value for small voltages. For $\left|e V_{d e v}\right|>\hbar \omega$ the noise increases linearly with the voltage and is due to shot noise. This transition from quantum to shot noise can directly be tested by measuring the detector current at a fixed level separation, $\epsilon=\hbar \omega$, as a function of the voltage across the QPC.
In Fig. 3b we plot $I_{\text {inel }}(\nu)$ for the same values of the total transmission. The main feature is an asymmetric broadening in the emission $(\nu>0)$ and absorption $(\nu<0)$ sides. We first consider the absorption side. For open channels $(D=1$ and $D=2)$, the nonequilibrium part of the noise is zero and no energy can be absorbed by the detector. For non-open channels $(D=1.5)$, the nonequilibrium noise is finite and the detector can absorb energy even at zero temperature; this is reflected as an inelastic current through the DQD for $\nu<0$. Emission is possible for both open and non-open channels due to $\mathrm{ZPF}$ so the inelastic current for $\nu>0$ is always finite.
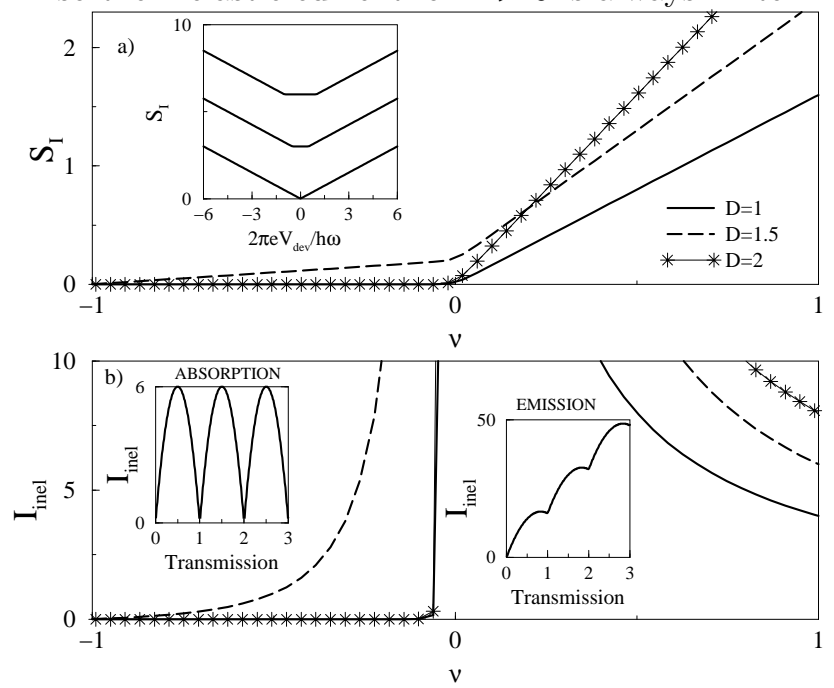

FIG. 3. (a) $S_{I}(\nu)$ (units of $\frac{4 e^{2}}{h} e V_{d e v}$ ) for different values of $D$. Inset: $S_{I}$ (units of $\frac{2 e^{2}}{\pi} \omega$ ) vs. $\frac{e V_{d e v}}{\hbar}$ (units of $\omega$ ) for different $\omega=1,0.5,0$, from top to bottom. (b) $I_{\text {inel }}(\nu)$ (units of $\left.32 \pi^{2} \kappa^{2} \frac{e}{h} \frac{T_{c}^{2}}{e V_{d e v}}\right)$. Insets: transmission dependence of $I_{\text {inel }}$ for a fixed value of the frequency; $\nu=-0.25$ for absorption and $\nu=0.25$ for emission.

The transmission dependence is shown in the insets to Fig. 3(b). At fixed $\nu$ the absorption oscillates as a function of $D$ (left inset) whereas the emission is an increasing function with plateau-like features (right inset).

To check the observability of these predictions we take as an example $D=1.5, \nu=0.5, \kappa=10^{-2}$ and $T_{c}=10 \mu \mathrm{eV}$. This implies, e.g. for the absorption side $I_{\text {inel }} \simeq 12 p \mathrm{~A}$ at $\epsilon / \mathrm{h}=1.22 \mathrm{GHz}(\mathrm{eV}$ dev $=10 \mu \mathrm{eV})$ For $\epsilon / h=12.17 G H z\left(e V_{\text {dev }}=100 \mu \mathrm{eV}\right), I_{\text {inel }} \simeq 1.2 p A$. These values are well within the resolution limits of present day techniques. 3, 20

A log-log plot of $I_{\text {inel }}$ vs. $\nu$ (Fig 4) demonstrates the transition from quantum to shot noise. At $\nu=-1$ (absorption), a sharp decline of the current marks such a transition. For open channels, the current on the emission side follows a power law behaviour indicating the occurence of an infrared divergence (see below).

We now examine the validity of our previous results. For non-open channels, $J(t)$ behaves for long times as: 


$$
J(t \rightarrow \infty) \simeq-8 \pi^{2} \kappa^{2} \sum_{m}^{N} D_{m}\left(1-D_{m}\right)\left|\frac{e V_{d e v}}{\hbar} t\right| .
$$

This linear time dependence coincides with previous results at equilibrium for an ohmic environment at finite temperature 10 12]. Eq. 8 defines two regimes: (a) $\nu>\nu_{c}$ with $\nu_{c}=8 \pi^{2} \kappa^{2} \sum_{m}^{N} D_{m}\left(1-D_{m}\right)$, the expansion for $J(t)$ in Eqs. 5-6 is valid. (b) $\nu<\nu_{c}$, the expansion for $J(t)$ cannot be used. Here, we obtain from Eq. 8: $I_{\text {inel }}(\epsilon) \simeq \frac{e T_{c}^{2}}{\pi \hbar} \frac{\eta}{\eta^{2}+\epsilon^{2}}$ with $\eta=e V_{d e v} \nu_{c} / 8$ [21]. Our results are within the limits of validity of regime (a).

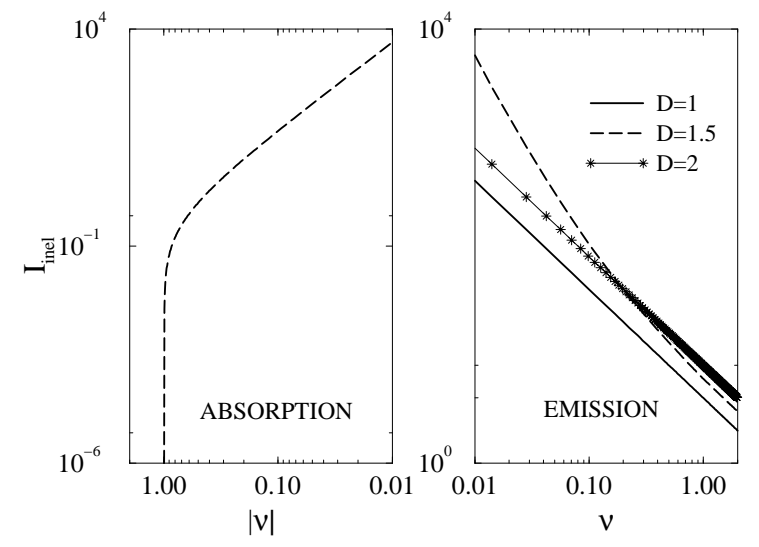

FIG. 4. $I_{\text {inel }}(\nu)$ vs. $\nu$ (log-log). Same units as Fig. $3 \mathrm{~b}$.

Note that for open channels $J(t \rightarrow \infty) \simeq$ $-\lambda\left\{\ln \left|\frac{e V_{\text {dev }}}{\hbar} t\right|+\xi+i \frac{\pi}{2}\right\}$ where $\xi=0.5772 \ldots$ is Euler's constant and $\lambda=8 \pi \kappa^{2} R_{K} G$. This behaviour leads to the infrared divergence $P(\epsilon) \simeq \epsilon^{\lambda-1}$ caused by the ZPF of the electron-hole pair excitations in the QPC. 22] In conclusion, the inelastic current through a DQD at low temperatures can provide a broad-band frequency resolved measurement of the equilibrium and non-equilibrium fluctuations in a nearby mesoscopic conductor. The asymmetry between absorption and emission processes gives a clear measurement of the nonequilibrium quantum noise. The predicted signal is well within the resolution limits of present day experiments on quantum dots [3] as well as on superconducting circuits 22. In the present case, the measurement by the DQD has a negligible effect on the transport through the QPC. If the QPC is replaced by a circuit in which superposition of quantum states is important (e.g. strongly coupled quantum dots), then our detection setup forms an interesting quantum measurement problem. 223]

We acknowledge Yuli Nazarov for fruitful criticism and discussions. We also thank Cees Harmans, Leonid Glazman, Wilfred van der Wiel, Toshimasa Fujisawa, Tjerk Oosterkamp, Michael Janus, Yann Kervennic, Silvano De Franceschi and Leonid Gurevich. Work supported by: the Dutch Organization for Fundamental Research on Mater (FOM), the NEDO joint research program
(NTDP-98), the EU via a TMR network, the NSF grant DMR 97-08499 and the MEC of Spain grant PF 9807497938.

[1] A. J. Leggett et al, Rev. Mod. Phys. 59, 1 (1987).

[2] Y. Nakamura et al, Nature, 398, 786 (1999).

[3] T. Fujisawa et al, Science 282, 932 (1998).

[4] Sh. Kogan, "Electronic noise and fluctuations in solids", Cambridge University Press 1996.

[5] M. J. M. de Jong and C. W. J. Beenakker in "Mesoscopic Electron Transport" (Kluwer, The Netherlands) 1997. Ed. by L.L. Sohn, L.P. Kouwenhoven and G. Schön.

[6] R. J. Schoelkopf et al, Phys. Rev. Lett. 78, 3370 (1997).

[7] E. Bucks et al, Nature 391, 871 (1998).

[8] T. H. Oosterkamp et al, Nature 395, 873 (1998).

[9] L. I. Glazman and K. A. Matveev, Sov. Phys. JETP 67, 1276 (1988).

[10] M. H. Devoret et al, Phys. Rev. Lett. 64, 1824 (1990).

[11] S. M. Girvin et al, Phys. Rev. Lett. 64, 3183 (1990).

[12] G. L. Ingold and Yu. V. Nazarov in "Single-Charge Tunneling" (Plenum, NY) 1992. Ed. by H. Grabert and M. H. Devoret.

[13] This is valid for gaussian fluctuations 12] provided the following realistic experimental conditions are met: a) weak coupling between detector and fluctuations and measurements in the linear response regime of the device. As long as tunneling events in the DQD weakly excite the environment only the power spectrum enters in $J(t)$. b) large level separation: for low frequencies a weakly perturbed environment is no longer well described by a set of harmonic oscillators.

[14] $J(t)$ depends on the order of the phase operators. In other words, the setup can distinguish between emission $(\omega>0)$ and absorption $(\omega<0)$, i.e, any noise spectrum entering in the measurable inelastic rate should obey $S_{I}(\omega)>S_{I}(-\omega)$. See C. W. Gardiner, "Quantum Noise", (Springer-Verlag) 1991, Chap. 1; for a discussion about symmetrization vs. non-symmetrization.

[15] Our formulation does not include other degrees of freedom of the environment (phonons, for example). At very low temperatures, and in the absence of any extra device, $I_{\text {inel }}(\epsilon>0)$ is finite due to spontaneous emission. [3] $I_{\text {inel }}(\epsilon<0)$ (absorption side), however, is virtually zero. This makes the absorption region ideal for measuring non-equilibrium fluctuations. The different sources of the noise in the emission side can be separated by studying the dependence on different parameters of the device.

[16] G. B. Lesovik, JETP Lett. 49, 592 (1989).

[17] S. E. Yang, Solid State Commun. 81, 375 (1992).

[18] M. Büttiker, Phys. Rev. B 45, 3807 (1992).

[19] H. B. Callen and T. A. Welton, Phys. Rev. 83, 34 (1951).

[20] $I_{\text {inel }}$ scales with $e V_{\text {dev }}$ at fixed $\nu$, which can be used to distinguish from heating effects.

[21] These regimes have been obtained in a different context by J. Siewert, Y. V. Nazarov and G. Falci, Europhys. 
Lett. 38, 365 (1997).

[22] Note the analogy of this case with the x-ray problem.

[23] See for example: S. A. Gurvitz, quant-ph/9806050. 